\title{
MODIFICATION DE LA SURVIE ET DE LA RÉCEPTIVITÉ CHEZ LE MOLLUSQUE, HOTE INTERMÉDIAIRE DE SCHISTOSOMA MANSONI PAR INOCULATION D'HÉMOLYMPHE PROVENANT DE MOLLUSQUES SENSIBILISÉS OU NON
}

\author{
G. MOLGEOT*, G. COLTRIS**, F. I.ANCASTRE*, J. P. OLAGHIISSI* \\ et J. BOL,OGNINI-TRENEY*
}

RESUMÉ. La survie et la réceptivité des Biomphalaria glabrala parasités par Schislosoma mansoni, ont été appréciées après inoculation d'hémolymphe saine ou sensibilisée à $S$. $m$.

l.'inoculation d'hémolymphe saine entraine une réceptivité élevée tandis que l'hémolymphe prólevée chez des $B . g$. parasités entraine une protection relative qui suggère un transfert d'immunité.

Mots-clés : Biomphalaria glabrata. Schistosoma mansoni. Hémolymphe. Immunité.

Survivorship and receptivity changes in the snail, intermediate host of Schistosoma
mansoni, by inoculation of

SUMMARY. The survival and receptivity of $B . g \cdot$ parasitized by $S . m$. were analysed after normal or sensibilized $S . m$. hemolymphe inoculation. The normal hemolymphe inoculation gives a high receptivity while the hemolymphe inoculation from parasitized $B . g$. gives a relative protection which suggests a transfert of immunity.

Key-lwords: Biomphalaria glabrata. Schistosoma mansoni. Hemolymph. Immunity.

Les mécanismes de défense actuellement décrits chez les mollusques sont des phénomènes de nature cellulaire. Ces mécanismes sont la phagocytose, l'encapsulement, la métaplasie, la nacréisation et le rejet de greffe. L'existence d'une immunité de type humoral, bien que soupçonnée, n’est pas prouvée (Benex et coll., 1980; Cheng T. C., 1979 ; Lackie A. M., 1980 ; Stumpf .J. L. et coll., 1980).

Dans des travaux antérieurs, nous avions montré que des cellules de l'hémolymphe de Biomphalaria glabrata parasitées par Schistosoma mansoni étaient

* Laboratoire de Parasilologie.

** Service de Biophysique, Facullé de Wédecine Saint-Antoine, 27, rue de Chaligny, F 75571 paris Cedex 12.

Accepté le 27 octobre 1986. 
capables de reconnaître la structure de Schistosoma mansoni (Golvan Y. J. et Mougeot G., 1973) ; d'autre part, depuis 1974, nous avons mis en évidence l'émission rythmique de furcocercaires chez le mollusque-hôte (Mougeot G. et coll., 1976).

Puisque les mollusques sont capables d'acquérir une immunité cellulaire et peut-être humorale (Tripp, 1975), nous nous sommes proposés d'en effectuer le transfert passif. Dans ce travail, nous avons inoculé de l'hémolymphe de mollusques parasités à des animaux sains, puis réalisé une infestation expérimentale par Schistosoma mansoni. L'appréciation des modifications engendrées par cette inoculation a été effectuée sur l'étude de la survie des mollusques et sur celle des émissions larvaires par rapport à deux lots témoins.

\section{Matériels et méthodes}

Dans cette expérimentation, nous avons utilisé une souche brésilienne de Schistosoma mansoni $(\mathrm{Sm})$ et une variété albinos de Biomphalaria glabrata $(\mathrm{Bg})$ de mème origine géographique. Les deux souches sont entretenues au laboratoire depuis une vingtaine d'années.

\section{1 - SCHÉMA EXPÉRIMENTAL}

Les mollusques ont été répartis en quatre groupes : deux groupes ont subi une injection d'hémolymphe et deux groupes servent de témoins suivant le protocole indiqué au tableaul $I$; les nombres de mollusques donnés en colonne 2 correspondent aux nombres de mollusques initiaux dans chaque lot (l'absence d'intervention préalable a permis de constituer un groupe d'animaux plus nombreux pour le lot $\left.n^{0} 1\right)$.

Tableau I. - Traitements subis par les différents lots de Bg avant infestation miracidiale.

\begin{tabular}{lcl}
\hline Lot & Nombre de mollusques & Traitement avant infestation \\
\hline 1 & 107 & Aucun \\
\hline 2 & 80 & $50 \mu \mathrm{l} \mathrm{NaCl} 0,1 \mathrm{M}$ \\
\hline 3 & 68 & $50 \mu \mathrm{l}$ d'hémolymphe de Bg sains \\
\hline 4 & 80 & $50 \mu \mathrm{l}$ d'hémolymphe de Bg infestés \\
\hline
\end{tabular}

Après un délai de 48 heures, les Bg des quatre lols sont soumis à une infestation par 5 à 6 miracidium de Sm. La survie et la production de furcocercaires (pourcentage d'infestations réussies et nombre de furcocercaires/mollusque) sont notées de la $4^{\mathrm{e}}$ (début des émissions de furcocercaires) à la $13^{\mathrm{e}}$ semaine suivant les injec- 
tions (le taux de survie au-delà de cette date étant trop faible pour permettre une étude statistique des résultats).

\section{2 -.. RecueIL DE L'HÉMOLYMPHE}

Deux pools d'hémolymphe ont été constitués par ponction intracardiaque, soit de Bg sains pour le premier pool, soit de Bg infestés six semaines au préalable par Sm pour le second pool. Après prélèvement, l'hémolymphe est conservée à $+4^{\circ} \mathrm{C}$ et utilisée pour les injections dans un délai de 1 heure au maximum.

\section{3 -- Michoinjections}

Après séchage soigneux sur papier-filtre, le mollusque est placé sous microscope stéréoscopique, la coquille est perforée à l'aide d'un scalpel et un fragment est enlevé au niveau de la zone péricardiaque. Une injection de $50 \mu l$ d'hémolymphe (lots 3 et 4 ) est pratiquée à ce niveau à l'aide d'une microseringue*.

Les mollusques témoins du lot 2 ont subi la même intervention mais ont été injectés avec une solution saline $(\mathrm{NaCl} 0,1 \mathrm{M})$. Les mollusques témoins du premier groupe ne subissent ni intervention ni injection.

\section{4 - InFestation Des MollusQues par "S. Mansoni "}

48 heures après les microinjections, les mollusques sont placés dans des borels contenant de l'eau vieillie où ils sont mis en contact individuellement avec 5 à 6 miracidiums de Sm pendant 24 heures. Ils sont ensuite placés dans des bacs et séjourneront dans une chambre humide à température de $25^{\circ} \mathrm{C}$. Ils sont nourris avec de la laitue fraîche.

5 - Mesure de la Survie

Nous avons noté à chaque semaine, de la $4^{\mathrm{e}}$ à la $13^{\mathrm{e}}$ semaine suivant l'infestation par Sm, ceux des mollusques encore en vie afin d'établir la statistique comparative entre les différents lots.

\section{6 -- Mesure du parasitisme}

Chez les animaux ayant survécu au moins jusqu'à la $4^{\mathrm{e}}$ semaine après l'infestation miracidiale, la présence de furcocercaires a été systématiquement recherchée hebdomadairement jusqu'à la $13^{\mathrm{e}}$ semaine (ou jusqu'à leur mort s'ils sont décédés avant la $13^{\mathrm{e}}$ semaine). L'étude a été faite suivant une modalité précédemment décrite (Mougeot G., 1976). Le recueil de ces données permet l'étude du parasitisme, d'établir les comparaisons entre les différents lots.

* Microseringue Hamilton 1725 I.T équipée d'une aiguille $731 \mathrm{KF}$. 


\section{7 -- Analyse statistique}

L'objet de l'étude étant la comparaison de la survie dans les quatre lots (et non l'étude de la survie dans un lot particulier), une étude globale concernant les quatre groupes simultanément a été pratiquée par test de chi-2 à chaque semaine sur le nombre de mollusques vivants et morts (les valeurs de chi-2 sont donc à interpréter en prenant trois degrés de liberté (ddl)). Les résultats sont représentés sur la figure 1. Les pourcentages de survie sont donnés avec les limites de confiance à $95 \%$ (moyenne \pm 2 écarts-types; lorsque le nombre de survivants est trop faible pour appliquer la formule de l'écart-type d'un pourcentage de distribution normale, le domaine de confiance à $95 \%$ a été calculé à partir de la distribution binomiale).

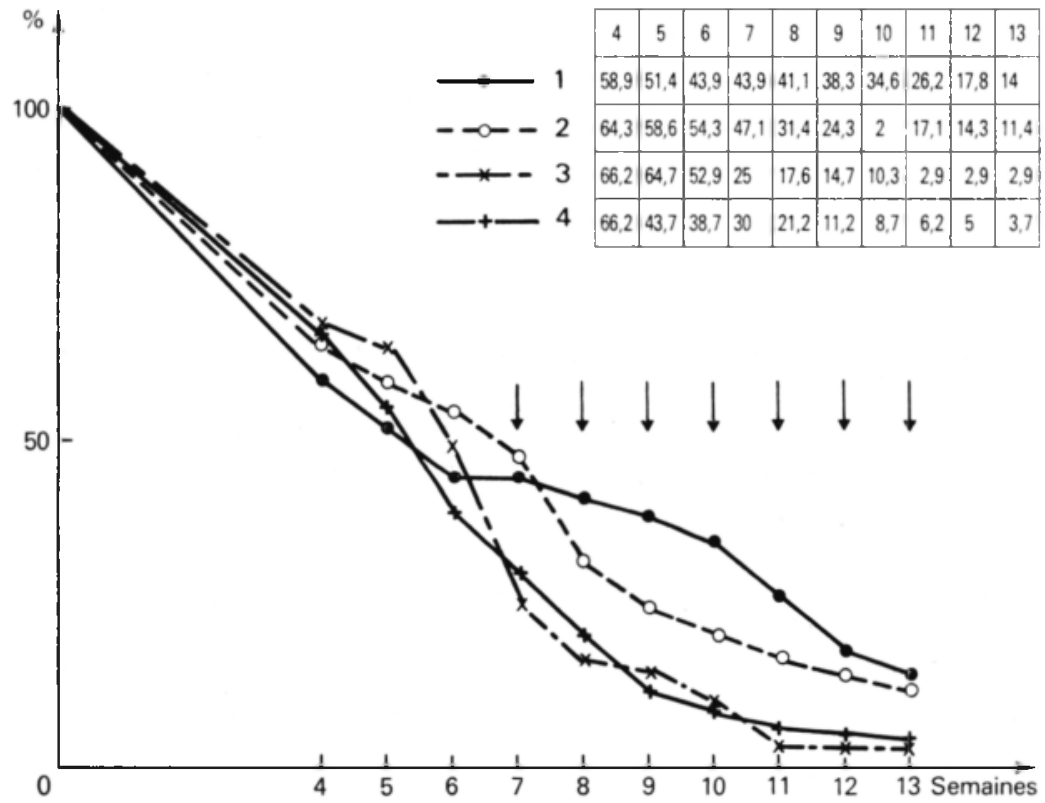

Fig. 1. Pourcentage de survie des mollusques (une flèche indique les différences significatives).

Lorsque l'étude globale (et seulement dans ce cas) montre une différence significative entre les lots, l'origine de cette différence a été recherchée par des comparaisons lot à lot. Nous avons ainsi comparé tant la mortalité cumulée que la mortalité "instantanée " par quinzaine.

Dans chaque lot, les planorbes ayant émis au moins une fois des furcocercaires ont été dénombrées ; les fréquences ont été comparées par test de chi-?. 


\section{Résultats}

1 - Étude et comparaison de la survif

Avant la $7^{\mathrm{e}}$ semaine, aucune différence significative n'existe entre les quatre lots; par contre, de la $7^{\mathrm{e}}$ semaine à la fin de l'étude, on note une différence significative de la survie entre les différents lots. L'analyse de données montre que cette différence ne peut s'expliquer par une différence entre les lots 3 et 4 (le test de chi-2 n'est jamais significatif entre ces deux lots). Quant aux lots témoins (1 et 2), l'incidence de l'intervention apparaît relativement mineure : ils ne diffèrent que transitoirement à la dixième semaine (chi-2 = 4,385; $\mathrm{P}<0,05)$ : cette légère différence à la $10^{\mathrm{e}}$ semaine ne suffit aucunement à expliquer à elle seule la différence très hautement significative entre les 4 lots à cette date $(\mathrm{P}<0,001)$ et peut être interprétée comme accidentelle (les deux lots ne manifestant qu'une seule semaine sur dix une différence significative au niveau de risque $5 \%$, on ne peut affirmer de différence significative entre ces deux groupes). Ceci justifie, vis-à-vis de l'étude de la survie, de réunir en un seul groupe témoin les lots 1 et 2 , et en un seul groupe traité les lots 3 et 4 ; la comparaison de ces 2 groupes établit le bien-fondé de ce regroupement en retrouvant les différences de survie entre témoins et traités aux mèmes semaines que précédemment (fig. 2 ).

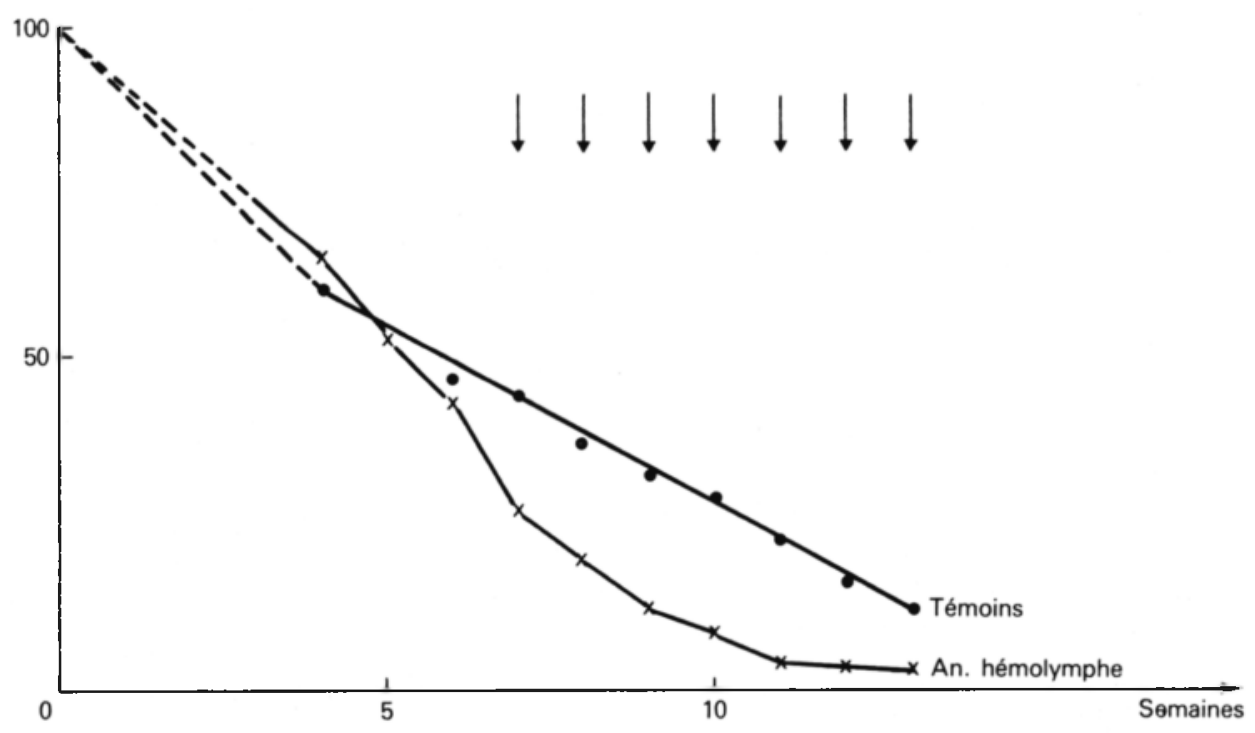

FIG. 2. - Courbe de survie.

(- - Témoins ; $(\mathbf{x}-\mathbf{x})$ animaux ayant reçu de l'hémolymphe. Les flèches $\left(\begin{array}{l}1 \\ \checkmark\end{array}\right)$ indiquent les temps où une différence significative est observée. 
La mortalité hebdomadaire des animaux témoins a été comparée à celle des animaux injectés : chez les animaux ayant reçu de l'hémolymphe, la mortalité est significativement plus élevée au cours des $7 \mathrm{e}$ semaine (chi-2 $=25,02$; $\mathrm{P}<0,001$ ), 9e semaine (chi-2 $=6,56 ; \mathrm{P}<0,02$ ), $11^{\mathrm{e}}$ semaine (chi-2 $=4,43$; $\mathrm{P}<0,05)$. L'absence de différence significative à la $8^{\mathrm{e}}$ et la $10^{\mathrm{e}}$ semaine semble due aux effectifs relativement faibles de planorbes décédés à chaque semaine. Pour y remédier, la mortalité peut être calculée sur 2 semaines consécutives ; la surmortalité des animaux traités par hémolymphe, apparue au cours des $6^{\mathrm{e}}$ et $7^{\mathrm{e}}$ semaines, reste constamment significative jusqu'aux $10^{\mathrm{e}}-11^{\mathrm{e}}$ semaines (fig. 3). Au-delà de la $11^{\mathrm{e}}$ semaine, la mortalité par quinzaine ne peut être comparée de façon ralide en raison d'effectifs trop faibles.

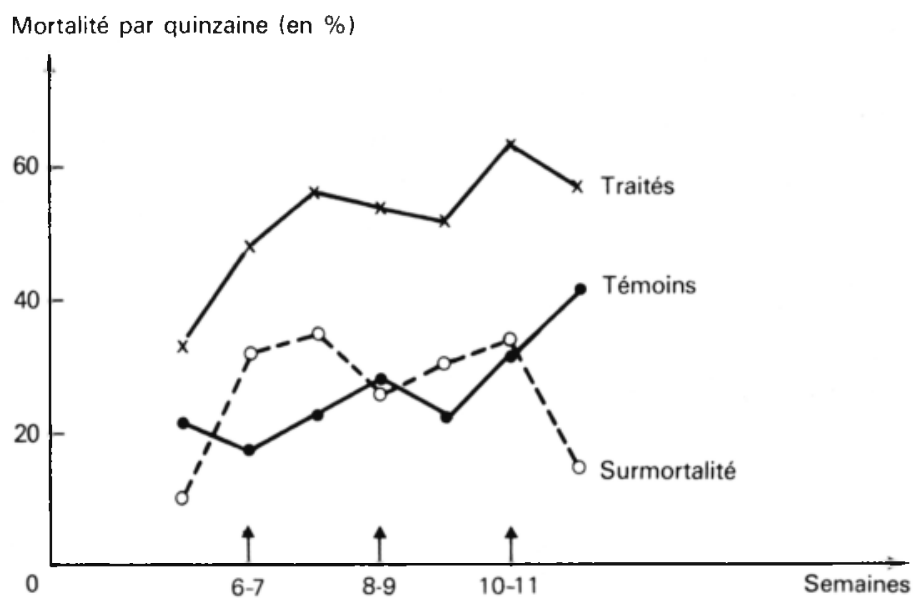

Fig. 3. - Mortalité comparée par quinzaine des témoins (-_-), des animaux traités par hémolymphe $(\mathrm{x}-\mathrm{x})$, surmortalité $(0-0)$ des animaux traités par raplort aux témoins (une flèche indique chaque intervalle où une surmortalité significative est observée).

\section{2 - Comparaison de la réceptivité des mollusques a "S. Mansoni »}

Nous avons étudié, chez tous les mollusques ayant survécu au moins jusqu'à la $4^{\mathrm{e}}$ semaine après l'infestation miracidiale, la fréquence de ceux ayant permis le développement normal de Sm jusqu'au stade de furcocercaires. L'étude a été renouvelée hebdomadairement de la $4^{\mathrm{e}}$ semaine à la $13^{\mathrm{e}}$; nous avons considéré comme "positifs" les mollusques ayant permis au moins une fois l'émission de furcocercaires (tableau $I I$ ).

L'étude globale montre une différence hautement significative (chi- $2=21,4$ avec $3 \mathrm{ddl} ; \mathrm{P}<0,001)$. Cette différence est liée à une positivité d'infestation significativement plus grande dans le lot 3 (mollusques ayant reçu de l'hémolymphe de $\mathrm{Bg}$ sains) que dans les autres lots (chi-2 $=16,76 ; \mathrm{P}<0,001$ ) ; il n'existe par contre pas de différence significative entre les lots 1,2 et 4 . 
L'injection d'hémolymphe saine provoque donc, dans notre expérience, une augmentation très importante de la réceptivité à $S$. mansoni ; par contre, l'injection d'hémolymphe sensibilisée ne favorise pas cette infestation.

Tableau II. - Nombre et pourcentage de mollusques ayant émis des furcocercaires dans chaque lot.

\begin{tabular}{|c|c|c|c|}
\hline Lots & $\begin{array}{c}\text { Nombre } \\
\text { de mollusques } \\
\text { vivants } \\
\text { à la } 4^{\mathrm{e}} \text { semaine }\end{array}$ & $\begin{array}{l}\text { Nombre } \\
\text { de mollusques } \\
\text { ayant émis des } \\
\text { furcocercaires }\end{array}$ & $\begin{array}{l}\% \text { de mollusques infestés } \\
\text { par rapport au nombre } \\
\text { de mollusques vivants } \\
\text { à la } 4^{\mathrm{e}} \text { semaine }\end{array}$ \\
\hline $\begin{array}{c}\text { Lot } 1 \\
107 \text { mollusques }\end{array}$ & 63 & 43 & 68 \\
\hline $\begin{array}{c}\text { Lot } 2 \\
\text { témoin saline } \\
70 \text { mollusques }\end{array}$ & 45 & 35 & 78 \\
\hline $\begin{array}{c}\text { Lot } 3 \\
\text { hémolymphe } \\
\text { saine } \\
68 \text { mollusques }\end{array}$ & 45 & 44 & 98 \\
\hline $\begin{array}{l}\text { Lot } 4 \\
\text { hémolymphe } \\
\text { sensibilisée } \\
80 \text { mollusques }\end{array}$ & 53 & 31 & 58 \\
\hline
\end{tabular}

\section{Discussion}

Dans cette série d'expériences, nous avons soumis des Biomphalaria glabrala à l'infestation par $S$. mansoni après un prétraitement consistant à administrer de l'hémolymphe, provenant de mollusques de même souche (sains ou parasités); nous avons pu constater certains effets entraînés par cette administration.

1) L'administration d'hémolymphe à des B. glabrala, avant infestation miracidiale, entraîne chez ces animaux une surmortalité par rapport aux animaux témoins n'ayant pas reçu d'hémolymphe. Cette surmortalité n'apparaît pas liée à l'acte opératoire comme le montre l'absence de différence entre les 2 lots témoins mais bien à l'administration du produit biologique.

Cet effet de l'hémolymphe est différé, apparaissant dans ces expériences brutalement au cours de la $7^{\mathrm{e}}$ semaine ; la mortalité cumulée demeure ultérieurement anormalement élevée. La surmortalité hebdomadaire, apparue brutalement à la $7^{\mathrm{e}}$ semaine, persiste au-delà (sur tout le temps où les effectifs concernés sont suffisants pour la mesurer). La surmortalité due à l'hémolymphe n'est pas significativement différente suivant l'origine du produit (hémolymphe de Bg sains ou au contraire infestés) alors mème que cette origine modifie les résultats de l'infestation. 
2) Le taux de survie à la $4^{\mathrm{e}}$ semaine ne présente pas de différence significative parmi les 4 lots : les pourcentages d'infestation réussie sont donc exprimés par rapport aux mollusques survivants à la $4 \mathrm{e}$ semaine dans le lot correspondant. L'étude globale montre une différence hautement significative entre les lots, dont l'origine s'est avérée être due à un taux d'infestation très supérieur dans le lot $n^{\circ} 3$; les trois autres lots ne présentant pas de différence significative entre eux. On ne peut exclure néanmoins que la surmortalité ultérieure (au-delà de la 7 e semaine) du groupe 4 par rapport aux groupes témoins ne masque une éventuelle surinfestation de ce lot; mais cette éventualité n'explique pas la différence d'infestation entre les lots 3 et 4 qui présentent une mortalité comparable tout au long de l'étude.

Plusieurs hypothèses permettent d'expliquer cette facilitation parasitaire (Voisin G. A. et coll., 1970) :

- la constitution antigénique de chaque planorbe est différente ; l'administration d'hémolymphe étrangère va mobiliser ces défenses (Michelson E., 1975; Tripp M. R., 1975) : ceci pourrait en diminuer l'efficacité vis-à-vis d'une infestation parasitaire ultérieure et expliquer l'augmentation de réceptivité observée dans le lot no 3 . La même hypothèse permettrait d'expliquer les résultats que nous avions obtenus (Mougeot G. et coll., 1979) lors d'infestations successives d'Angiostrongylus canlonensis puis de $S$. mansoni : le premier parasite qui pénètre favorise l'installation du second;

-- l'hémolymphe de certains mollusques posséderait une action de facilitation parasitaire ou bien une action immuno-suppressive.

L'introduction d'hémolymphe en provenance de mollusques parasités ne produit pas cette facilitation parasitaire : on peut supposer que l'hémolymphe de ces mollusques parasités contient des constituants cellulaires et/ou des composants solubles dirigés contre le parasite. Ces éléments transférés chez un mollusque receveur seront présents dès la pénétration du parasite et vont limiter le développement de ce dernier ; cette hypothèse est corroborée par les résultats de Golvan Y.J. et coll. (1973) : les granulocytes présents dans l'hémolymphe de Biomphalaria glabrala parasités par S. mansoni sont capables de reconnaître dans une forte proportion l'antigène de $\mathrm{Sm}$; les granulocytes de $\mathrm{Bg}$ non parasités ne le reconnaissent que rarement. Ces cellules seraient capables de transférer leur immunité.

La surmortalité très comparable des lots 3 et 4 ne saurait être complètement expliquée par l'infestation parasitaire très différente dans ces 2 lots : il ne saurait être exclu que le conflit immunitaire entraîné par l'administration d'hémolymphe étrangère puisse accentuer la mortalité des planorbes.

\section{Conclusion}

L'introduction d'hémolymphe totale, provenant de planorbes sensibilisées ou non, chez des Biomphalaria glabrata sains, soumis ensuite à l'infestation parasitaire par Schislosoma mansoni, entraîne des modifications de comportement vis- 
à-vis de ce parasite par rapport à des lots témoins. L'introduction d'hémolymphe saine a pour conséquence une meilleure infestation alors que l'introduction des composants de l'hémolymphe prélevée chez des Biomphalaria glabrala préalablement parasités par Schistosoma mansoni entraîne une protection relative, un taux de parasitisme faible. Ces modifications pourraient être en faveur d'un transfert d'immunité.

\section{BIBLIOGRAPHIE}

1. Benex J., Jacobelli G. : Exploration de l'immunilé chez les mollusques hôtes de Schistosomes : Biomphalaria glabrata. Bull. Soc. Pathol. Exot., 1980, 2, 206-213.

2. Cheng T. C. : Cellular immunity in molluses with emphasis on the intermediate host of human infecting Schistosomes. Nat. Acad. Sci. Ilar News, 1979, 22, 9-16.

3. Golvax Y. J., Mougeot G. : Mise en évidence des propriétés cytoadhérentes des cellules de l'hémolymphe de Biomphalaria glabrata parasité par Schistosoma mansoni. C. R. Acad. Sci. Paris, CCLXXVI (12-02-73), série D-1229.

4. LACKIE A. M. : Invertebrate Immunity. Parasitology, 1980, 80, 393-412.

5. Lancastre F., Coutris G., Bolognini-Treney J., Traore L., Mougeot G. : Schistosomose expérimentale. III. Observalions sur l'infestation de Biomphalaria glabrala par un miracidium de Schistosoma mansoni. Ann. Parasitol. Hum. et Comp., 1984, 59, 79-94.

6. Michelson E. H. : Cellular defense mechanisms and tissue alterations in gastropod molluscs. In: "Invertebrate immunity" (Moramo Rosch, Schupe R. E., Eds), Academic Press, New York, 1975, 201-223.

7. Mougeot G., Golvan Y. J. : 1) Étude des relations entre la longévité de Biomphalaria glabrata el son infestation par Schistosoma mansoni. 2) Ettude de la variation quantitative des furcocercaires. Bull. Soc. Sci. Vet. Med. Comp. Lyon, 1976, 2, 119-123.

8. Mougeot G., Golvan Y. J., Depernet D. : Interaction nématode-trématode chez Biomphalaria glabrata vecteur de la bilharziose humaine aux Antilles et en Amérique du Sud. Bull. Soc. Sci. Vet. Med. Comp. Lyon, 1979, 3, 151-156.

9. StumpF J. L., Gilbertson D. E. : Differencial leukocytic response of Biomphalaria glabrata so infection with Schistosoma mansoni. J. Invert. P'athol., 1980, 35, 217-218.

10. Thipp M. R. : Humoral factors and molluscan immunity. In: "Invertebrate immunity " (Moramorosch K., Shope R. E., Eds). Academic Press, New York, 1975, 201-223.

11. Vorsix G. A. : Immunily and Lolerance. A unified concept. Cell. Immunol., 1971, 2, 670. 